Carta al Editor

\section{Comparación de las sentencias de incapacidad permanente por fibromialgia en España: diferencias según la resolución sea favorable para el paciente o para el Instituto Nacional de la Seguridad Social}

Comparison of rulings on permanent disability due to fibromyalgia in Spain: differences according to whether the resolution is favorable to the patient or to the National Institute of Social Security

\section{Sr. Editor:}

La fibromialgia (FM) es un trastorno músculo-esquelético crónico de causa desconocida y evolución variable que origina grados variables de discapacidad con un claro impacto sobre la calidad de vida ${ }^{1}$. Su elevada prevalencia en edades productivas implica importantes repercusiones laborales. Aunque, los datos de discapacidad laboral varían considerablemente dependiendo de la población estudiada, la mayoría de los estudios estiman que entre el 25 y $50 \%$ de pacientes $^{2-5}$ debe dejar de trabajar por su enfermedad.

En España, se reconoce la incapacidad temporal ${ }^{5}$ con una duración máxima de doce meses, prorrogables por otros seis, cuando se presuma que durante ellos el trabajador puede ser dado de alta médica por curación, situación que normalmente no se da en estos pacientes. Cuando se agota este plazo, el INSS, a través de los equipos de valoración de incapacidades, puede reconocer la situación de prórroga con un límite de seis meses más o bien, iniciar un expediente de incapacidad permanente (IP). En el caso de que la valoración sea aceptada, el proceso termina y el paciente obtiene la incapacidad, pero si es rechazada, puede interponer una reclamación contra el dictamen del equipo de valoración de incapacidades ante el propio INSS. Si es desestimada por el INSS, el paciente puede presentar una demanda ante el Juzgado de lo Social y, en su caso, recurrir ante el correspondiente Tribunal Superior de Justicia (TSJ). En la actualidad, se desconoce el porcentaje de propuestas presentadas por pacientes con FM que son aceptadas por el equipo de valoración de incapacidades o por el Juzgado de lo Social como IP, pero sí se puede acceder a las que son presentadas ante el TSJ. En este trabajo se analizan las variables asociadas al reconocimiento de la FM como causa de IP a la luz de las sentencias dictadas en suplicación por los TSJ.

Se han analizado todas las sentencias presentadas ante los TSJ españoles por este motivo (1978-2008) utilizando como fuente la base de datos Westlaw, que contiene toda la jurisprudencia a texto completo ${ }^{6}$. Se trata de una base de datos de acceso restringido a los usuarios e instituciones que están suscritos. Se ha utilizado la palabra clave «fibromialgia».

Se identificaron 148 sentencias, de estas, 9 se excluyeron por no contener la información completa. Noventa y siete se presentaron ante el TSJ de Murcia, 9 en Cataluña, 6 en Baleares y 6 en Asturias y el resto repartidas entre las diferentes comunidades autónomas. La tabla 1 presenta las variables en función de si la resolución de la sentencia fue favorable para el paciente o para el INSS. Como se observa, la descripción en la sentencia de la presencia de puntos dolorosos o gatillo es la única variable que muestra diferencias significativas para decantar el fallo a favor del paciente. Las diferencias entre las proporciones se calcularon mediante la prueba de la $\mathrm{x}^{2}$, estableciéndose el nivel de significación estadística en $\mathrm{p}=0,005$.

Un bajo nivel educativo y socioeconómico, presencia de otras enfermedades como el lupus o la depresión o trabajos en ocupaciones que requieren esfuerzo físico son los determinantes más consistentemente asociados a la incapacidad temporal en la FM en investigaciones realizadas en servicios asistenciales ${ }^{3,7,8}$. Sin embargo, al analizarlas, la única característica que se relaciona

\section{Tabla 1}

Distribución del resultado de las sentencias presentadas ante los Tribunales Superiores de Justicia de España $(1978-2008)$ solicitando la concesión de incapacidad permanente

\begin{tabular}{|c|c|c|c|c|c|c|}
\hline & \multicolumn{2}{|c|}{$\begin{array}{l}\text { Sentencias } \\
\text { favorables } \\
\text { para INSS }\end{array}$} & \multicolumn{2}{|c|}{$\begin{array}{l}\text { Sentencias } \\
\text { favorables } \\
\text { para paciente }\end{array}$} & \multirow[t]{2}{*}{ Total } & \multirow[t]{2}{*}{ Valor $\mathrm{p}$} \\
\hline & $\mathrm{n}$ & $\%$ & $\mathrm{n}$ & $\%$ & & \\
\hline & 104 & & 35 & & 139 & \\
\hline \multicolumn{7}{|l|}{ Sexo } \\
\hline Mujer & 93 & 89,4 & 33 & 94,3 & 126 & 0,39 \\
\hline Hombre & 11 & 10,6 & 2 & 5,7 & 13 & \\
\hline \multicolumn{7}{|l|}{ Edad } \\
\hline $30-45$ & 64 & 61,5 & 18 & 51,4 & 82 & 0,35 \\
\hline$>45$ & 40 & 38,46 & 17 & 48,5 & 57 & \\
\hline \multicolumn{7}{|l|}{ Ocupación } \\
\hline Administración & 13 & 12,5 & 1 & 2,9 & 14 & 0,32 \\
\hline Comercio & 13 & 12,5 & 60 & 17,1 & 73 & \\
\hline Servicios & 45 & 43,3 & 20 & 57,1 & 65 & \\
\hline Agricultura & 30 & 28,8 & 7 & 20 & 37 & \\
\hline Manuales y construcción & 3 & 2,9 & 1 & 2,9 & 4 & \\
\hline \multicolumn{7}{|l|}{ Tiempo de evolución } \\
\hline$>3$ & 82 & 78,8 & 26 & 74,3 & 108 & 0,57 \\
\hline$<3$ & 22 & 21,2 & 9 & 25,7 & 31 & \\
\hline \multicolumn{7}{|l|}{ Diagnóstico único } \\
\hline Sí & 6 & 78,8 & 5 & 74,3 & 11 & 0,10 \\
\hline No & 98 & 21,2 & 30 & 25,7 & 128 & \\
\hline \multicolumn{7}{|l|}{ Puntos gatillo } \\
\hline Sí & 7 & 6,7 & 7 & 20 & 14 & 0,02 \\
\hline No & 97 & 93,3 & 28 & 80 & 125 & \\
\hline \multicolumn{7}{|l|}{ Grado de incapacidad } \\
\hline Permanente parcial & & & 4 & 8,6 & 4 & \\
\hline Permanente total & & & 21 & 60 & 21 & \\
\hline Permanente absoluta & & & 8 & 22,9 & 8 & \\
\hline Gran invalidez & & & 3 & 8,6 & 3 & \\
\hline
\end{tabular}


con la concesión de la IP es la presencia de puntos específicos que a la presión desencadenen dolor. Un estudio realizado en Canadá, en el que se revisaban las 194 sentencias presentadas en sus tribunales entre 1986-2003 para evaluar el impacto en la percepción de los jueces en las reclamaciones por FM, señalaba que estos perciben la opinión de los especialistas como el argumento más creíble al tomar decisiones ${ }^{9}$.

\section{Conclusión}

El hecho de que se trate de una enfermedad caracterizada por ausencia de pruebas que indiquen de manera objetiva la severidad puede influir en que los jueces valoren la ausencia de este criterio diagnóstico como un elemento negativo para conceder la incapacidad.

\section{Bibliografía}

1. Wolfe F, Anderson J, Harkness RM, Bennett RM, Carp XJ, Goldenberg DL, et al. Health status and disease severity in fibromyalgia: results of a six center longitudinal study. Arthritis Rheum. 1997;40:1571-9.

2. Wilson HD, Robinson JP, Turk DC. Toward the identification of symptom patterns in people with fibromyalgia. Arthritis Rheum. 2009;61:527-34.

3. Salido M, Navarro P, Judez E, Hortal R. Factores relacionados con la incapacidad temporal en pacientes con fibromialgia. Reumatol Clin. 2007;3:67-72.
4. Al -Allaf AW. Work disability and health system utilization in patients with fibromyalgia syndrome. J Clin Rheumatol. 2007;13:199-201.

5. Ley General de la Seguridad Social Española. Real Decreto legislativo 1/1994 de 20 de Junio. BOE $\mathrm{n}^{\circ} 154$ de 29 de junio.

6. Servicio Jurídico en línea de editorial Aranzadi [consultado 28/9/2008] Disponible en: http://www.westlaw.es. [Acceso 28 Septiembre de 2008].

7. Lawrence RC, Hochberg MC, Kelsey JL. Estimates of prevalence of selected arthritis and musculoskeletal diseases in the United States. J Rheumatol 1989;16:427-41.

8. Al Dhanhani AM, Gignac MA, Su J, Fortin PR. Work disability in systemic lupus erythematosus. Arthritis Rheum. 2009;61:378-85.

9. Le Page JA, Iverson GL, Collins P. The impact of judge's perceptions of credibility in fibromyalgia claims. Int J Law Psychiatry. 2008;31:30-4.

Juan Carlos Restrepo Medrano ${ }^{\mathrm{a}, \mathrm{b}, *}$, Elena Ronda-Pérez ${ }^{\mathrm{b}}$, Carmen Vives-Cases ${ }^{\mathrm{b}}$, Diana Gil-González ${ }^{\mathrm{b}}$ y Fernando BallesterLaguna $^{c}$

a Facultad de Enfermería, Universidad de Antioquía, Medellín, Colombia

${ }^{\mathrm{b}}$ Área de Medicina Preventiva y Salud Pública, Universidad de Alicante, Alicante, España

${ }^{\mathrm{c}}$ Departamento de Derecho del Trabajo y Seguridad Social, Universidad de Alicante, Alicante, España

*Autor para correspondencia.

Correo electrónico: juance@tone.udea.edu.co

(J.C. Restrepo Medrano). 